\title{
Las trayectorias de crecimiento de la Argentina, el Brasil, Chile y México: una visión comparativa a través de la lente del espacio marco (framework space)
}

\author{
Carmem Feijo, Lionello Franco Punzo \\ y Marcos Tostes Lamônica
}

\section{Resumen}

En este artículo se analizan las diferentes trayectorias de crecimiento de cuatro economías latinoamericanas, a saber, la Argentina, el Brasil, Chile y México, comparando la fase de estrategias de crecimiento por sustitución de importaciones con el período más reciente de integración financiera en la economía mundial. La hipótesis de trabajo es que las diferentes trayectorias de crecimiento son el resultado de los vínculos entre las condiciones macroeconómicas y los cambios en las estructuras de producción. Se realiza un análisis basado en una herramienta metodológica que compara las estrategias de crecimiento con los modelos canónicos, la metodología del framework space (un espacio marco donde se representan sus "predicciones"), que sirve para comparar fases de crecimiento descritas como una combinación cambiante del dinámico perfil del crecimiento de la productividad (una condición del lado de la oferta) con el comportamiento de la acumulación de capital (una condición del lado de la demanda).

\section{Palabras clave}

Crecimiento económico, sustitución de importaciones, liberalización económica, integración económica, política económica, historia económica, análisis comparativo, Argentina, Brasil, Chile, México

Clasificación JEL

E44, O11, O54

Autores

Carmem Feijo es Profesora del Departamento de Economía de la Universidad Federal Fluminense (Brasil). Correo electrónico: cbfeijo@gmail.com.

Lionello Franco Punzo es Profesor del Departamento de Economía y Estadística de la Universidad de Siena (Italia). Correo electrónico: punzo@unisi.it.

Marcos Tostes Lamônica es Profesor Asociado de la Universidad Federal Fluminense (Brasil). Correo electrónico: marcostostes@hotmail.com. 


\section{Introducción}

Hasta finales de la década de 1970, la industrialización por sustitución de importaciones dirigida por el Estado fue la estrategia de desarrollo predominante en la mayoría de las economías latinoamericanas. Esta estrategia se abandonó tras la crisis de la deuda externa de los años ochenta, de la que se tardó casi toda la década en recuperarse. El fin de la prolongada recesión económica que siguió a la crisis de la deuda (conocida como la "década perdida") se asocia con la apertura económica y a la profundización del proceso de financierización en la región 1 .

Como parte del proceso de financierización, la liberalización del comercio, las privatizaciones y la desregulación financiera se convirtieron en las principales recomendaciones de política económica, y la gestión de las políticas monetarias y fiscales pasó a estar en gran medida subordinada a los vaivenes de los mercados financieros internacionales. Como señalan Ocampo y Vos (2008), el espacio de políticas en las economías en desarrollo, en particular las latinoamericanas, se ha reducido mucho desde la apertura económica ${ }^{2}$. Según estos autores, esa reducción supuso una pérdida de autonomía para las autoridades económicas a la hora de implementar políticas macroeconómicas anticíclicas eficaces y coherentes con objetivos y políticas de desarrollo a más largo plazo (pág. 29) ${ }^{3}$. Su principal argumento es que, dado el carácter procíclico de los flujos de capital, la apertura económica limita la capacidad de las autoridades para gestionar la política económica anticíclica en respuesta a las fases de auge y depresión. De hecho, Ocampo (2007) sostiene que, en lugar de mitigar, los flujos de capital hacia los países en desarrollo exacerban tanto los auges como las recesiones económicas (pág. 9).

El interés en las estrategias de crecimiento de las economías latinoamericanas se ha renovado en los últimos años. Bárcena y Prado (2015), por ejemplo, presentan un análisis de las diferentes fases del crecimiento latinoamericano desde la década de 1980. A partir de los ciclos económicos de la región, en su mayoría determinados por choques externos, en el estudio se propone un criterio para determinar los diferentes períodos de crecimiento. Conforme la tradición estructuralista, se supone que el cambio estructural depende de la fuerza y la continuidad de la inversión en acumulación de capital, que es la principal fuerza que impulsa y sostiene el crecimiento. Dado que la inversión es el componente más dinámico de la demanda agregada, la gestión de la política macroeconómica a corto plazo, realizada con miras a un mayor crecimiento a largo plazo, debería frenar la volatilidad de los principales precios macroeconómicos y mantener una posición fiscal anticíclica, una tasa de inflación baja y estable a largo plazo, tasas de interés reales bajas y un tipo de cambio real competitivo a lo largo del tiempo.

Este trabajo se centra en las diferentes trayectorias de crecimiento de cuatro economías latinoamericanas, a saber, la Argentina, el Brasil, Chile y México, comparando el período de estrategias de crecimiento por sustitución de importaciones con el período más reciente de integración financiera en la economía mundial ${ }^{4}$. La hipótesis de trabajo es que las diferentes trayectorias de crecimiento son el resultado de los vínculos entre las condiciones macroeconómicas y los cambios en la estructura de producción. En este sentido, la política económica desempeña un papel importante en la explicación del proceso de crecimiento e influye así en la trayectoria a largo plazo. Cuando el espacio de políticas

1 En términos generales, se puede decir que la financierización tiene lugar cuando los mercados, los actores, las prácticas y las representaciones financieras tienen un impacto cada vez mayor en las estructuras y las dinámicas sociales (véase Epstein, 2005).

2 China y la India son ejemplos poco frecuentes de integración financiera prudente y no es casualidad que hayan obtenido mejores resultados que otras economías desarrolladas y en desarrollo desde la crisis financiera internacional. Véase un análisis del desempeño económico de Brasil, Rusia, India, China y Sudáfrica (BRICS) desde la crisis financiera en Nassif, Feijo y Araújo (2016).

3 Rey plantea otra manera de ver la pérdida de autonomía en las políticas económicas de los países en desarrollo integrados financieramente. En un influyente trabajo, la autora sostiene que las políticas monetarias independientes en las economías en desarrollo solo son posibles si se gestiona la cuenta de capital (Rey, 2015).

4 Véase, por ejemplo, Moreno y Pérez (2009), que mencionan que estas fueron las economías que siguieron una estrategia de industrialización dirigida por el Estado. También fueron responsables de más del 50\% del valor agregado manufacturero total en América Latina en la década de 2010. 
disminuye, los resultados de crecimiento a largo plazo se ven afectados y el cambio estructural no contribuye a aumentar el potencial de crecimiento. En otras palabras, se asume que el cambio estructural es importante para explicar los resultados de crecimiento a largo plazo, pero que no se produce sin complicaciones y, por lo general, da lugar a un crecimiento desequilibrado con consecuencias en los equilibrios internos y externos. Por lo tanto, para que el producto potencial aumente, es necesario ampliar el espacio de políticas de manera que las políticas a largo plazo (como las políticas industriales y tecnológicas) puedan coordinarse estrechamente con las políticas macroeconómicas a corto plazo 5 .

Para argumentar este punto, se realiza un análisis basado en la metodología del framework space, que permite comparar fases de crecimiento descritas como una combinación cambiante del dinámico perfil del crecimiento de la productividad (una condición del lado de la oferta) con el comportamiento de la acumulación de capital (una condición del lado de la demanda). La interacción entre estos dos factores genera naturalmente una trayectoria de crecimiento no lineal, interrumpida por saltos específicos o discontinuidades, y que por supuesto no necesariamente tiende a una posición de equilibrio predeterminada (conforme la teoría convencional). Una de las ventajas de la metodología del framework space es que permite interpretar las fases del crecimiento económico con referencia a la posición kaldoriana o bien a la neoschumpeteriana, y clasificar en consecuencia los diversos cambios estructurales experimentados por las economías latinoamericanas. Así, la principal contribución de este trabajo consiste en proporcionar una interpretación analítica, basada en la metodología del framework space, de las diferencias en las trayectorias de crecimiento de estas cuatro economías latinoamericanas.

El trabajo se divide en cinco secciones, incluida esta introducción. En la segunda sección se presenta la metodología del framework space. En la tercera sección se distinguen tres períodos asociados con diferentes trayectorias de crecimiento, que se analizan en la cuarta sección para cada una de las economías. La contribución de esta sección consiste en interpretar el historial de crecimiento de cada país sobre la base de las pruebas empíricas del framework space, proporcionando así una visión general de cada economía. A la luz del análisis comparativo del framework space, la principal conclusión es que la apertura económica de los años noventa no contribuyó a promover el proceso de convergencia económica en ninguna de las cuatro economías analizadas. En la quinta y última sección se resumen las principales conclusiones.

\section{La metodología del framework space}

El enfoque convencional del crecimiento supone implícitamente que las economías del mundo real tienden a una trayectoria particular a largo plazo que forma parte de un régimen estable, y que este es un factor de atracción tan fuerte que toda dinámica a más corto plazo es un movimiento pasajero y prácticamente irrelevante 6 . Sin embargo, los datos observados varían todo el tiempo, de manera que los patrones de crecimiento deberían evaluarse en función de la dinámica de las variables relacionadas. Para abordar este tema, la metodología del framework space incorpora una serie de modelos de crecimiento y es a partir de esta que se construyen los patrones reales de crecimiento?.

El framework space es un dispositivo analítico utilizado para centrarse en variables como la acumulación de capital, el empleo y la productividad. La principal justificación para elegirlas es, por

\footnotetext{
5 Siguiendo la tradición kaleckiana, Titelman y Pérez (2015, pág. 181) lo expresan de la siguiente manera: "Una primera implicación importante derivada del análisis es que la macroeconomía para el desarrollo no puede plantear una dicotomía entre ciclos y tendencias o entre el corto o el largo plazo. Las fluctuaciones a corto plazo definitivamente influyen en los resultados a largo plazo".

6 Esta predicción es muy clara en la teoría neoclásica, que sostiene que la tasa de crecimiento económico en el estado estacionario al que se llegue dependerá únicamente del crecimiento de la población y el progreso técnico. Fue esta claridad la que le dio importancia como teoría y como conjunto de proposiciones falsables econométricamente.

7 Véase una presentación y discusión exhaustiva del enfoque del framework space en Böhm y Punzo (1992, 1994 y 2001), Brida y Punzo (2003), Gaffard y Punzo (2005) y Feijo, Punzo y Lamônica (2012).
} 
supuesto, que son las variables empleadas en las teorías del crecimiento más conocidas. El framework space toma únicamente la tasa de crecimiento de la inversión por empleado (en la escala vertical) y la tasa de crecimiento de la productividad laboral (en la escala horizontal) (véase Böhm y Punzo, 2001, pág. 48). El objetivo de esta selección es explicar la relación entre la dinámica de las variaciones de la productividad y la dinámica de las variaciones de la inversión por empleado. La construcción del framework space parte de las series de producto interno bruto (PIB) o valor agregado $(v a)$, formación bruta de capital fijo real $(i)$ y empleo $(e)$. Así, se define como:

$$
\begin{aligned}
& \frac{d(\log V A-\log e)}{d t}=g v \\
& \frac{d(\log I-\log e)}{d t}=g i
\end{aligned}
$$

donde $g v$ es la tasa de crecimiento de la producción por empleado (una medida del crecimiento de la productividad) y gi es la tasa de crecimiento de la inversión, siempre por empleado. Las variables gv y gi proporcionan las coordenadas de la trayectoria dinámica de una economía determinada en el plano (véase el gráfico 1). Los cambios en los niveles relativos de las coordenadas ( $g v, g i$ representan cambios en la relación dinámica de la economía analizada y pueden significar una modificación en la intensidad de los cambios en las variables, o el régimen, es decir, un cambio estructural ${ }^{8}$.

\section{Gráfico 1}

Regímenes de crecimiento en el framework space

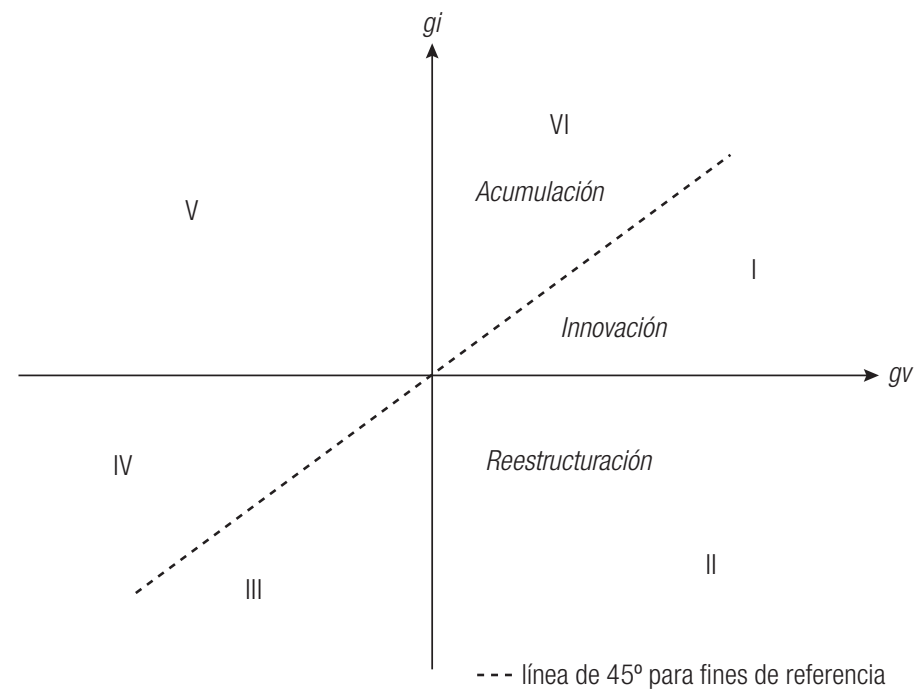

Fuente: Elaboración propia.

8 El cambio estructural se interpreta de forma diferente en otros enfoques. Puede producirse cuando hay cambios en la composición del PIB o de los agregados económicos, o cuando hay un cambio en la estructura organizativa e institucional de una economía. De acuerdo con Kaldor, el cambio estructural se observa cuando hay cambios en la composición de la industria manufacturera con respecto a la intensidad tecnológica, captada mediante las elasticidades de la demanda de exportaciones e importaciones. Así, según el modelo de Kaldor-Thirlwall, un cambio estructural puede ser favorable o desfavorable para el crecimiento de una economía en la que la balanza de pagos está en equilibrio. Véanse Dixon y Thirlwall (1975) y Thirlwall (1979). Asimismo, el cambio estructural implica una interrelación entre las fuerzas de la oferta y la demanda que explican las trayectorias de crecimiento. Una gran cantidad de estudios asociados con la teoría del crecimiento endógeno apoya este enfoque (De Long y Summers, 1991; León-Ledesma, 2002; Syverson, 2010). León-Ledesma (2002), por ejemplo, estimó un modelo estructural para un conjunto de países de la Organización de Cooperación y Desarrollo Económicos (OCDE) en el período 1965-1994. Además de los efectos de la inversión y de la ley de Kaldor-Verdoorn tradicional, en el trabajo también se captan los efectos directos e indirectos de la innovación y el progreso técnico en el comportamiento de la productividad laboral. En palabras del autor, la innovación no solo conduce a un mayor grado de diferenciación y calidad de los productos, sino también a la innovación de los procesos que conduce a un aumento de la productividad (León-Ledesma, 2002, pág. 204). 
En el gráfico 1 se muestra la manera de interpretar las trayectorias de crecimiento y sus fases en el sistema del framework space. En este marco se tratan seis regímenes de crecimiento estándar y un régimen de crecimiento especial. Este último es la línea que interseca el plano de las coordenadas ( gv, gi ) a 45 grados y corresponde a una generalización del llamado corredor harrodiano que separa el régimen VI por encima de la línea del régimen I por debajo de la línea9. Tanto el régimen VI (acumulación) como el régimen I (innovación) se encuentran en el primer cuadrante, donde se produce el crecimiento económico, es decir, donde las tasas de crecimiento de la productividad ( $g v$ ) y la inversión por empleado ( $g i$ ) son ambas positivas. El régimen II (reestructuración), en el segundo cuadrante, combina un $g v$ positivo y un gi negativo. Los demás regímenes (III, IV y V) se tratan, en la estructura analítica del framework space, como reflejos de los regímenes mencionados anteriormente.

La interpretación relevante para el argumento de este trabajo es que el framework space está dotado de tres categorías (o clases de modelos) de regímenes de crecimiento, a saber: i) del estado estacionario; ii) de la acumulación, en el que la atención se centra en los cambios en la intensidad de la inversión y la productividad aumenta con la acumulación de capital (suponiendo que se incorpore el progreso técnico) ${ }^{10}$; y iii) de la innovación tratada, en principio, como funcionalmente independiente de la acumulación de capital productivo. En este caso el crecimiento se explica por las innovaciones, ya sean nuevas formas organizativas o procesos o la introducción de nuevos productos que aumentan la brecha entre los costos unitarios y el precio final. La trayectoria de crecimiento está dada por la secuencia formada como una función de los pares de $g v$ y gi, distribuidos en el plano del framework space. Cada punto del framework space en el que se intersecan estas coordenadas está asociado con una trayectoria de crecimiento; de una parte a la otra, cambia la clase generadora del crecimiento.

El framework space puede relacionarse directamente con las predicciones de las teorías convencionales. Por ejemplo, la teoría neoclásica identifica un factor de atracción global único, una trayectoria de estado (cuasi) estacionario en que la tasa de crecimiento $g_{N C}$ se define como ${ }^{11}$ :

$$
g_{N C}=n+\lambda
$$

donde $n$ es la tasa de crecimiento de la población y $\lambda$ es el progreso técnico. Sin embargo, la tasa de crecimiento económico observada $g$ por lo general será diferente de este crecimiento exógeno. Así, el framework space trata de explicar la tasa de crecimiento endógeno $g_{E N}$ como la desviación de la tasa de crecimiento en estado estacionario ${ }^{12}$.

$$
g_{E N}=g-(n+\lambda)
$$

El framework space toma la tasa $g_{N C}$ como punto de partida del gráfico 1, es decir, las coordenadas $(0,0)$, para realizar el análisis de la tasa de crecimiento endógeno. Así, se supone que

9 El comportamiento harrodiano está representado por las trayectorias de estado estacionario. Las coordenadas $(0,0)$ están asociadas con la trayectoria de crecimiento exógeno (Böhm y Punzo, 2001, pág. 53) con una tasa de progreso tecnológico nula (véase más adelante).

10 Véanse Kaldor (1957) y Kaldor y Mirrlees (1962), entre otros.

${ }^{11}$ Cabe señalar que en la teoría neoclásica no hay una distinción clara entre regímenes de crecimiento de acumulación y de innovación cuando se supone que el progreso técnico es exógeno. Para incorporar el progreso técnico en la teoría del crecimiento moderna, se modifican los factores de producción del capital $(K)$ y el trabajo $(L)$, y la función de producción agregada tradicional $Y=Y(K, L)$ puede escribirse añadiendo un multiplicador dependiente del tiempo $A(t)$ que incorpora el progreso técnico global. Así, de acuerdo con Romer (2012, pág. 10), la función de producción agregada neoclásica que incorpora el progreso técnico puede escribirse como: $Y(t)=F(K(t), A(t) L(t)$ ), donde t denota el tiempo. Sin embargo, según Aghion y Howitt (2009), esta modificación de la función de producción tradicional para incorporar el progreso técnico sigue sin explicar la manera en que este se incorpora. Por lo tanto, $A(t)$ puede considerarse un dispositivo de modelización útil, pero que tiene poco poder explicativo.

12 León-Ledesma y Thirlwall (2002) probaron con éxito la hipótesis de la endogeneidad de la tasa de crecimiento natural para un grupo de 15 países de la OCDE, al igual que Libânio (2009) para el grupo de las 12 mayores economías de América Latina. La tasa de crecimiento natural aumenta en los períodos de expansión y disminuye en los períodos de contracción porque la fuerza de trabajo y el crecimiento de la productividad son elásticos con respecto al crecimiento de la demanda y la producción. 
el comportamiento de la tasa efectiva $g$ influye en la trayectoria a largo plazo y, por lo tanto, la tasa de crecimiento endógeno puede explicarse mediante dos regímenes o modelos de crecimiento: la acumulación de capital y la innovación.

En resumen, todas las teorías a largo plazo están presentes en el framework space bidimensional. El modelo de Solow (1957) es el punto de partida y los puntos observados que se encuentran en otros lugares se asocian con el crecimiento endógeno, que puede explicarse tanto por la teoría, es decir, la acumulación de capital, como por el régimen de innovación ${ }^{13}$.

\section{Trayectorias de crecimiento en el período 1951-2014: una visión general de los resultados de crecimiento de las cuatro economías seleccionadas}

Para arrojar luz sobre la manera de determinar las distintas fases que pueden estar asociadas con distintos regímenes de crecimiento en las economías seleccionadas, se recurre en primer lugar a algunos indicadores relacionados con la evolución del sector manufacturero.

En la tradición kaldoriana, el desarrollo no es neutro desde el punto de vista sectorial y se asigna un papel especial a la industria manufacturera en el impulso y el mantenimiento de las tasas de crecimiento a largo plazo. En el cuadro 1 se presentan algunos indicadores de la evolución de la proporción de valor agregado manufacturero y del comercio internacional de manufacturas a lo largo del tiempo.

Cuadro 1

Argentina, Brasil, Chile y México: indicadores seleccionados del sector manufacturero, años seleccionados

(En porcentajes)

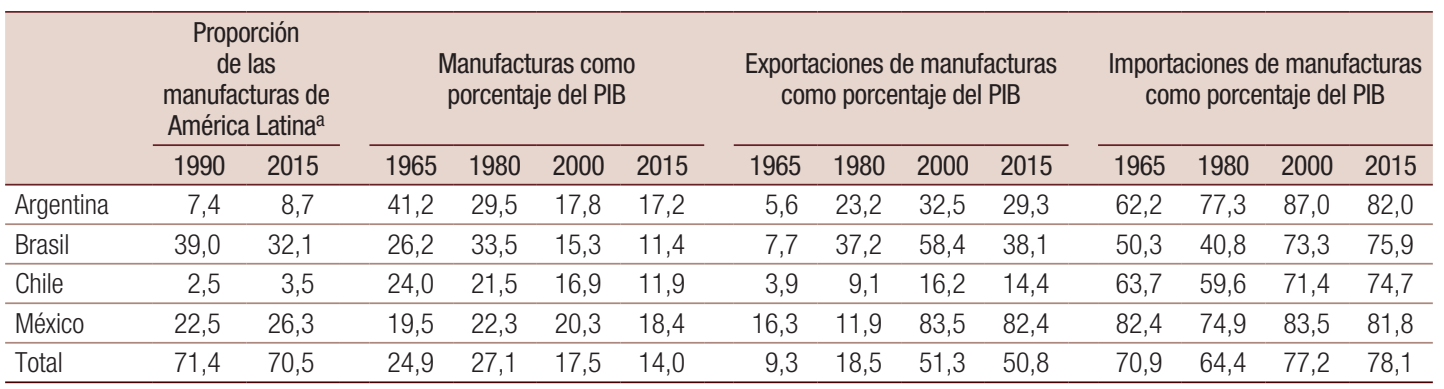

Fuente: Banco Mundial, Indicadores del Desarrollo Mundial [base de datos en línea] https://datacatalog.worldbank.org/dataset/ world-development-indicators.

a Valor agregado manufacturero en dólares constantes de 2010.

En las dos primeras columnas (las estimaciones para el Brasil están disponibles a partir de 1990) se muestra la contribución relativa de cada país al total de la industria manufacturera en América Latina. Aunque la participación del Brasil disminuyó en las décadas de 1990 y 2000, sigue siendo la economía más industrializada de la región.

En las cuatro columnas siguientes se presenta la proporción del PIB de la industria manufacturera de cada país y de toda la región. La tendencia a la industrialización se muestra en la última fila, la parte

\footnotetext{
${ }^{13}$ Los modelos endógenos con énfasis en las situaciones de desequilibrio se inspiran en las contribuciones de Richard Goodwin. Véase Punzo (2006).
} 
del PIB correspondiente a la industria manufacturera. En la región en su conjunto, esta aumentó entre 1965 y 1980 y disminuyó posteriormente. A nivel de países, se observa un aumento en el Brasil y México en el período mencionado, seguido por una disminución en todos los países latinoamericanos seleccionados en las décadas siguientes. En los primeros años de la década de 1980 se produjeron profundos cambios en la trayectoria de crecimiento de las economías latinoamericanas, que resultaron sumamente afectadas por la crisis de la deuda.

En las ocho columnas restantes se presenta la evolución de la proporción de productos manufacturados en el total de las exportaciones e importaciones. Esta aumentó en todas las economías seleccionadas desde la década de 1960 hasta el año 2000. La participación de las manufacturas en las exportaciones mexicanas disminuyó entre 1965 y 1980, pero mostró un incremento significativo en 2000, tras la firma del Tratado de Libre Comercio de América del Norte (TLCAN) en 1994. La proporción de exportaciones de manufacturas disminuyó en todas las economías entre 2000 y 2015. Sin embargo, en comparación con 1965, se observa que seguía siendo significativamente mayor en este último año, sobre todo como resultado del proceso de industrialización. En general, al considerar las fechas de inicio y fin del período examinado, la proporción de importaciones de manufacturas también aumentó. Sin embargo, entre 1965 y 1980 disminuyó en todas partes, excepto en la Argentina.

Entre 1980 y 2000, las importaciones de manufacturas aumentaron en líneas generales, siguiendo el movimiento análogo de las exportaciones. En el período de 2000 a 2015, mientras la proporción de exportaciones de manufacturas se redujo, la proporción de importaciones de manufacturas aumentó en todas partes, excepto en la Argentina. Por lo tanto, en lo que respecta a los flujos de comercio de productos manufacturados, el período de 1980 a 2000 muestra un cambio significativo en la balanza comercial de las economías de la región, que podría apuntar a un cambio significativo también en el régimen de crecimiento.

En el cuadro 2 se presentan las tasas de crecimiento del PIB de las economías examinadas en períodos de tiempo seleccionados. Estos se han elegido para captar diferentes trayectorias de crecimiento y períodos de transición para un único indicador, la tasa media de crecimiento del PIB en el intervalo de tiempo (la fase). En el cuadro 3 se proporciona un resumen de las principales características de cada una de las fases.

\section{Cuadro 2}

Argentina, Brasil, Chile y México: tasa de crecimiento del producto interno bruto (PIB) ${ }^{\mathrm{a}}$, períodos seleccionados

(En porcentajes)

\begin{tabular}{lccc}
\hline & Fase 1 (1951-1981) & Fase 2 (1982-1999) & Fase 3 (2000-2014) \\
\hline Argentina & 2,9 & 2,1 & 3,3 \\
\hline Brasil & 7,0 & 2,3 & 3,3 \\
\hline Chile & 3,6 & 4,6 & 4,2 \\
\hline México & 6,6 & 2,1 & 2,5 \\
\hline
\end{tabular}

Fuente: Universidad de Groninga, Penn World Tables 9.0 [base de datos en línea] https://www.rug.nl/ggdc/ productivity/pwt/pwt-releases/pwt9.0?lang=en.

a PIB real a precios constantes en millones de dólares de 2011.

\section{Cuadro 3}

Fases de crecimiento económico propuestas

\begin{tabular}{lll}
\hline & Período & Descripción \\
\hline Fase 1 & $1951-1981$ & $\begin{array}{l}\text { Régimen de crecimiento basado en la industrialización por } \\
\text { sustitución de importaciones dirigida por el Estado }\end{array}$ \\
\hline Fase 2 & $1982-1999$ & Crisis de la deuda y consolidación de la apertura económica \\
\hline Fase 3 & 2000-2014 & $\begin{array}{l}\text { Régimen de crecimiento basado en la integración } \\
\text { económica en un mundo asimétrico }\end{array}$ \\
\hline
\end{tabular}

Fuente: Elaboración propia. 
La fase 1, que corresponde al período de industrialización dirigida por el Estado, capta el período más dinámico del Brasil y México, los países más industrializados de la región. La fase 2 abarca la "década perdida", que afectó a la mayoría de las economías latinoamericanas, y el período de mayor inestabilidad en los mercados externos, debido a las crisis asiática y rusa de los años noventa. En el pasaje de la fase 1 a la fase 2, el espacio de políticas se redujo en la mayoría de las economías debido, entre otras cosas, a la escasez de liquidez internacional para las economías muy endeudadas. En consecuencia, la fase 2 se considera como una transición a un nuevo régimen de crecimiento. La fase 3, por otra parte, se caracteriza por la consolidación de este nuevo régimen de crecimiento, marcado por una mayor integración financiera y comercial ${ }^{14}$. Así, las fases implican diferentes modelos de crecimiento.

En resumen, al considerar el período global, las dos fases principales son la primera y la tercera, mientras la segunda representa una transición. La fase de industrialización por sustitución de importaciones dirigida por el Estado se caracterizó por la industrialización como motor del desarrollo. La intervención del Estado en diferentes ámbitos de la actividad económica fue el principal factor que impulsó las decisiones de inversión y el desarrollo tuvo una fuerte orientación hacia el mercado interno. Sin embargo, esta rápida industrialización provocó desequilibrios externos que culminaron en la crisis de la deuda externa. La apertura económica fue la estrategia para superar la escasez de liquidez externa. El período de transición se caracterizó por las reformas estructurales y de mercado que tuvieron lugar sobre todo en las décadas de 1980 y 1990, según el consenso de Washington. Estas se caracterizaron por la liberalización que desencadenó las fuerzas del mercado, consideradas como la forma más eficiente de asignar los recursos. Esta fase contrasta fuertemente con la orientación de la primera fase, pues el Estado y otras instituciones ajenas al mercado se consideraban una solución "de segundo orden". La tercera fase fue la de un nuevo régimen de crecimiento en el que las economías estaban más integradas entre sí y a nivel mundial, pero también eran más vulnerables a los choques externos.

\section{Interpretación de las trayectorias de crecimiento en el framework space}

Un régimen de crecimiento identifica cualitativamente una dinámica de crecimiento específica, generada por un modelo de crecimiento determinado. Por lo tanto, mientras una trayectoria es toda secuencia genérica de caminos de crecimiento, una dinámica de régimen de crecimiento es la representación de las características cualitativas de una trayectoria destiladas mediante las propiedades de la representación de su régimen (Böhm y Punzo, 1992 y 1994; Brida y Punzo, 2003). Así, en el framework space, la trayectoria real de una economía (su experiencia de crecimiento histórica) está secuenciada como una cadena de caminos de crecimiento dentro de un régimen, entre regímenes, o ambos. Cuando los caminos de crecimiento abarcan más de un régimen, se habla de un cambio de régimen como un cambio cualitativo en el modelo de crecimiento, y esta discontinuidad se entiende como una manifestación de un cambio estructural subyacente, que se explicita o "emerge" mediante ciertos aspectos cualitativos de la dinámica observada de la economía. Combinando las nociones implícitas en la metodología del framework space con la segmentación predeterminada en fases históricas, se obtiene una variedad de posibles formas de explicar las trayectorias de crecimiento a largo plazo.

\footnotetext{
${ }^{14}$ La tercera fase se caracterizó por una mayor integración financiera de las economías. Contrariamente a los supuestos de la teoría neoclásica, no se observó un cambio estructural hacia sectores de producción más diversificados y avanzados tecnológicamente. De hecho, como ha demostrado Amsdem (2001, pág. 85), las políticas de libre comercio como estrategia de convergencia económica parecen haberse limitado a Suiza y Hong Kong (Región Administrativa Especial de China). Chang (2003, pág. 2) también destacó que la mayoría de los países desarrollados adoptaron políticas industriales y comerciales consideradas malas en la evaluación de la corriente neoclásica, como la protección de la industria naciente y las subvenciones a la exportación.
} 
En este trabajo se desea examinar la combinación de trayectorias de crecimiento cualitativamente distintas, la dinámica del régimen. Utilizando el framework space es posible analizar las trayectorias de crecimiento de las cuatro economías seleccionadas bajo el supuesto (introducido a continuación) de que en gran medida operaban bajo las mismas condiciones económicas externas. Así, las diferentes trayectorias de los países podrían interpretarse como respuestas específicas a las medidas de gestión de la política macroeconómica nacional que repercuten en los resultados de la productividad y la inversión. En el gráfico 2 se presenta la trayectoria de las cuatro economías examinadas (Argentina, Brasil, Chile y México) en las fases de crecimiento predefinidas. En el cuadro 4 se resumen sus trayectorias de crecimiento vistas a través del instrumento del framework space ${ }^{15}$.

\section{Gráfico 2}

Argentina, Brasil, Chile y México: diagramas de framework space, 1951-2014

\section{A. Argentina}

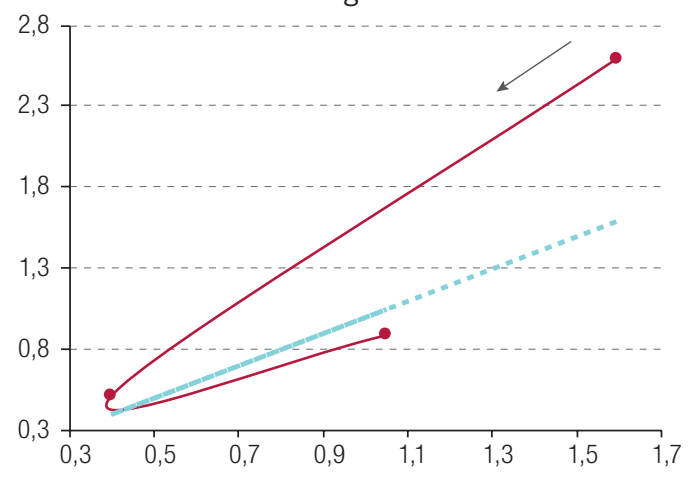

C. Chile

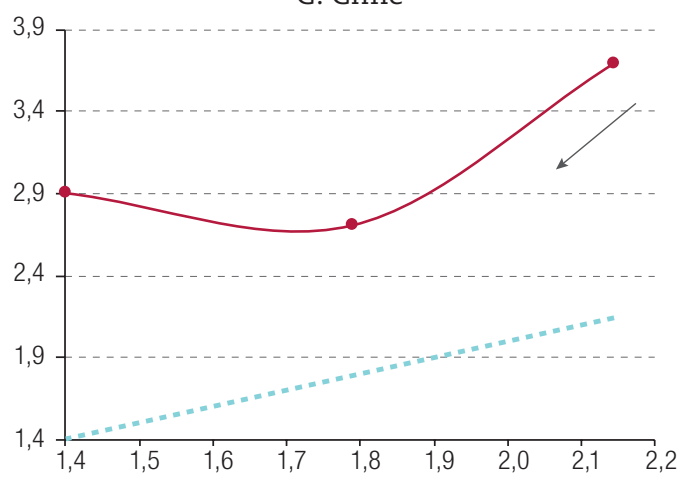

B. Brasil

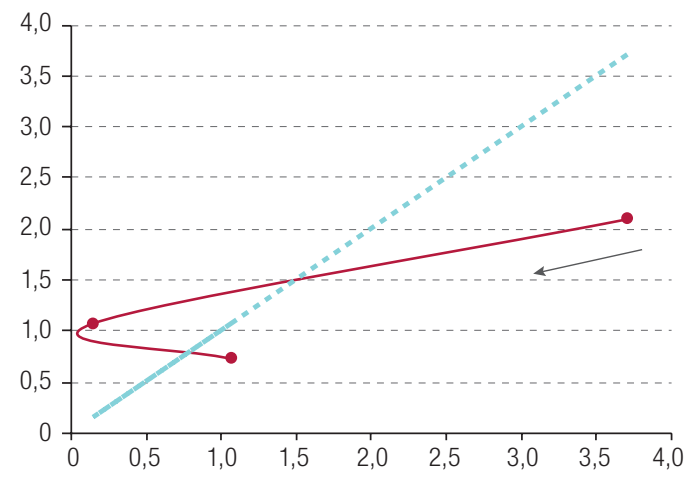

D. México

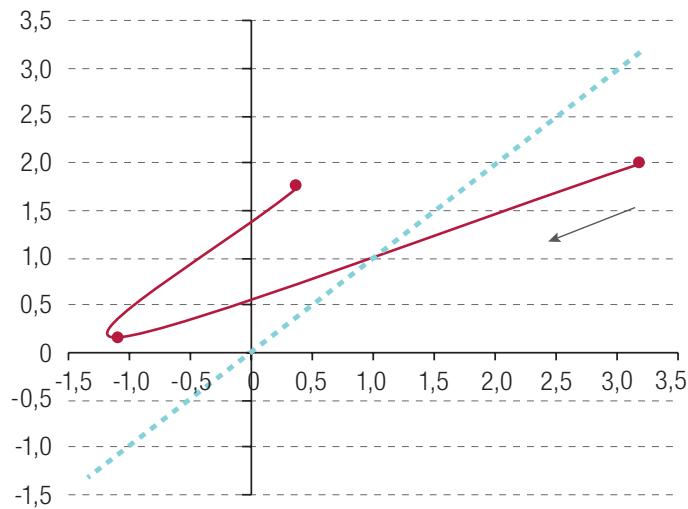

$\rightarrow(g v, g i)==$ = línea de $45^{\circ}$ para fines de referencia

Fuente: Elaboración propia.

Nota: Véase la explicación de los términos en el anexo A1.

a Comienza en 1952.

Cuadro 4

Argentina, Brasil, Chile y México: resumen de las trayectorias de crecimiento

\begin{tabular}{lcccc}
\hline & Argentina & Brasil & Chile & México \\
\hline Fase 1 (1951-1981) & Acumulación & Innovación & Acumulación & Innovación \\
\hline Fase 2 (1982-1999) & Acumulación & Acumulación & Acumulación & Reducción \\
\hline Fase 3 (2000-2014) & Innovación & Innovación & Acumulación & Acumulación \\
\hline
\end{tabular}

Fuente: Elaboración propia.

15 Véase una descripción de las variables del gráfico 2 en el anexo A1. 


\section{Fase 1: régimen de crecimiento basado en la industrialización por sustitución de importaciones dirigida por el Estado (1951-1981)}

Después de la Segunda Guerra Mundial, los grandes países de América Latina se embarcaron en un proceso de industrialización basado en la sustitución de importaciones en la industria y en un estricto control de los tipos de cambio ${ }^{16}$. Se estimuló el endeudamiento externo, que se tradujo en altas tasas de inversión. Los años sesenta y setenta suelen considerarse una edad de oro en la historia económica de América Latina ${ }^{17}$.

Como régimen de crecimiento, la industrialización dirigida por el Estado se centró en un conjunto de acuerdos institucionales destinados a promover el cambio estructural con miras a alcanzar más rápidamente a las economías maduras. El Brasil y México constituyen ejemplos exitosos de la aplicación de estrategias de industrialización por sustitución de importaciones. Como se puede observar en el gráfico 2, en esta fase se registraron, en promedio, las mayores tasas de crecimiento de la productividad para estas economías. En ambos casos, la trayectoria de crecimiento se sitúa en el cuadrante de la innovación, con un crecimiento medio de la productividad agregada que supera el crecimiento medio de la inversión por trabajador durante el período. Este resultado puede considerarse como una clara indicación de que el aumento de la productividad agregada fue consecuencia de la intensificación del proceso de industrialización. En ambos países, la industrialización se basó en políticas proteccionistas, es decir, en cada etapa del proceso de sustitución de importaciones, los gobiernos señalaron determinadas industrias como prioritarias para la política industrial y utilizaron tanto licencias de importación como aranceles elevados para proteger el sector manufacturero ${ }^{18}$.

El proceso de industrialización argentino siguió un camino diferente. El sector agrícola presenta ventajas comparativas porque la Argentina está dotada de grandes extensiones de tierra fértil. Así, la industrialización supuso el desplazamiento del centro dinámico de las actividades agrícolas y ganaderas hacia las manufactureras. La industrialización terminó en 1976 (véanse Ferrer, 2004; Câmara y Vernengo, 2013), cuando se impuso un programa de política económica neoliberal que dejó el proceso inconcluso. Entre 1951 y 1981, la trayectoria de crecimiento de la Argentina se sitúa en el cuadrante de la acumulación (véase el gráfico 2). El rasgo más llamativo del patrón de desarrollo argentino desde la Segunda Guerra Mundial no es tanto la industrialización dirigida por el Estado como la inestabilidad política que caracterizó el desarrollo del país (véase Câmara y Vernengo, 2013, págs. 115-116). De hecho, el golpe militar de 1976 fue un intento de hacer volver a la Argentina al modelo de crecimiento agroexportador económicamente liberal del "pasado glorioso" del país (Ferrari y Cunha, 2008, pág. 27). Se utilizaron métodos ortodoxos en un intento de frenar la inflación que aumentaba constantemente ${ }^{19}$ y a finales de la década la economía se había estancado20.

El patrón de crecimiento chileno en el período 1952-1981 también se sitúa en el cuadrante de la acumulación. Esto indica que el cambio estructural promovido por la estrategia de industrialización

${ }^{16}$ La industrialización basada en políticas proteccionistas que favorecían a las industrias pesadas nacientes recibió un fuerte impulso en la década de 1950 y el modelo centro-periferia de Prebisch tuvo una marcada influencia.

17 Véase, por ejemplo, Bértola y Ocampo (2012).

${ }^{18}$ En el Brasil, la política industrial fomentó activamente la industrialización y se pusieron en marcha planes nacionales de desarrollo para hacer frente a los grandes desequilibrios en la balanza comercial, sobre todo en la década de 1970. La industrialización brasileña dependía en gran medida del ahorro externo. Paradójicamente, las crisis de la balanza de pagos reforzaron los argumentos del gobierno a favor de renovar el uso de instrumentos proteccionistas y la sustitución de importaciones. La estrategia de sustitución de importaciones seguida por México, que también se basaba en políticas proteccionistas, siguió un camino diferente a partir de los años sesenta, y el régimen proteccionista se basó cada vez más en las licencias de importación y menos en la protección arancelaria. Según Ros (1993), el criterio esencial para la concesión de licencias de importación era la disponibilidad de suministros nacionales.

${ }^{19}$ En 1978, el plan implementado por el ministro neoliberal José Alfredo Martínez de Hoz fracasó por completo y se produjo una crisis bancaria.

20 Antes de la moratoria mexicana, la Argentina tenía la mayor relación entre la deuda externa y el PIB de América Latina. 
por sustitución de importaciones no modificó las características más importantes de la economía, incluida su marcada dependencia de la extracción de minerales. De hecho, la orientación estatal de la economía chilena llegó a todos los sectores económicos. Entre 1964 y 1973, Chile implementó un proceso de reforma agraria que afectó al 50\% de las tierras agrícolas. En 1971, se nacionalizó toda la riqueza mineral y se creó la Corporación Nacional del Cobre de Chile (CODELCO), que se convirtió en el mayor exportador del país, posición que mantiene hasta el día de hoy. Si bien los mercados financieros fueron regulados por el Estado, el golpe militar de 1973 llevó la economía a un programa neoliberal radical, que redujo rápidamente la presencia del Estado mediante un amplio programa de privatizaciones que abarcó las empresas públicas, los bancos e incluso la seguridad social, el fomento de los seguros de salud privados y la expansión de la educación privada. La apertura económica supuso la internacionalización del sector financiero. Estas reformas económicas expusieron la economía a los movimientos de los mercados financieros internacionales y, en consecuencia, la suspensión de pagos de México en 1981 afectó gravemente el PIB chileno, que se redujo más de un 10\% en $1982^{21}$.

En el Brasil, la estrategia de industrialización por sustitución de importaciones fue prácticamente abandonada tras el impago externo de México en 1982. De hecho, este tipo de estrategias dirigidas por el Estado acabaron por abandonarse en la mayoría de los países latinoamericanos, pues su principal defecto era que se basaban en la existencia de déficits externos y en el recurso a cantidades cada vez mayores de préstamos externos, una estrategia insostenible debido principalmente a la elevada volatilidad asociada con la financiación externa. En consecuencia, su abandono fue el resultado del comportamiento de las balanzas comercial y de capitales, pues esto significaba que la inversión, la principal variable a la hora de incrementar la renta y el producto globales, era insostenible. Una crítica frecuente a la estrategia de sustitución de importaciones es que dejaba poco margen para el crecimiento basado en las exportaciones, pues el excesivo proteccionismo generaba ineficiencias en la producción industrial. La falta de dinamismo de los ingresos por exportación se convirtió en un importante cuello de botella para la industrialización por sustitución de importaciones, pues el sector industrial era intensivo en importaciones (Sapelli, 2003).

La edad de oro de las economías latinoamericanas, durante la cual el cambio estructural favoreció la industrialización, llegó a su fin con la moratoria externa de México.

\section{Fase 2: crisis de la deuda, consolidación de la apertura económica y transición a un nuevo régimen de crecimiento (1982-1999)}

El virtual abandono de la agenda desarrollista que guio las estrategias de crecimiento de América Latina después de la Segunda Guerra Mundial fue el resultado de la crisis de la deuda externa ${ }^{22}$. El aumento de la deuda externa tras el marcado incremento de las tasas de interés internacionales condujo a la implementación de políticas recesivas en busca de ajustes externos en todos los países endeudados. Las fuertes devaluaciones de las monedas nacionales tras las crisis de la balanza de pagos provocaron un aumento de los precios internos. En pocas palabras, una vez abandonadas las políticas desarrollistas, el espacio de políticas se redujo considerablemente en las economías latinoamericanas.

De acuerdo con el framework space (véase el gráfico 2), todas las economías entraron en un proceso de reducción de las tasas de crecimiento, tanto de la productividad como de la inversión por trabajador durante la segunda fase.

\footnotetext{
${ }^{21}$ De acuerdo con los Indicadores del Desarrollo Mundial, en dólares de 2005 en paridad del poder adquisitivo (PPA).

22 Moreno y Pérez (2009, pág. 37) afirman que, en la década de 1980, la crisis de la deuda que provocó la mayor disminución del crecimiento del producto en la historia de la región y afectó a la mayoría de los países latinoamericanos se utilizó como leitmotiv para realizar una crítica demoledora de las políticas de desarrollo anteriores y recomendar políticas basadas en el mantra "estabilizar, privatizar y liberalizar".
} 
Tras la moratoria externa mexicana de 1982, y a medida que aumentaba la fragilidad financiera del sector público, la inflación brasileña se consolidaba. Las altas tasas de inflación dominaron la situación macroeconómica a mediados de los años ochenta y principios de los noventa, período en el que se aplicaron diversos planes antiinflacionistas, con escaso o ningún éxito. Al mismo tiempo, las estrategias de desarrollo perdieron terreno en el debate económico, pues la renegociación de la deuda externa se convirtió en la mayor prioridad de la política económica. La alta inflación fue finalmente derrotada con el Plan Real de 1994. A principios de los años noventa se introdujeron reformas de liberalización del comercio, relativamente tarde en comparación con las otras economías seleccionadas. Sin embargo, se implementaron muy rápidamente: entre 1988 y 1994, se eliminó la mayoría de las barreras no comerciales y el arancel de importación nominal se redujo del 39,6\% al 11,2\% (promedio simple), mientras la desviación estándar disminuyó del 14,6\% al 5,9\% (Kume, Piani y de Souza, 2003, pág. 11). Sin embargo, de todas las reformas económicas adoptadas en el Brasil, la apertura de la cuenta de capital a corto plazo fue probablemente la principal responsable de exponer la economía nacional a la inestabilidad de la economía mundial y también de reducir la contribución de las políticas monetaria, fiscal y cambiaria al mantenimiento del crecimiento. Si bien, por una parte, la apertura de la economía ayudó a estabilizar la inflación crónica, por otra contribuyó al surgimiento de una nueva tendencia cíclica de apreciación de la moneda en términos reales que aumentó la vulnerabilidad de la economía a los choques externos. Por último, la integración financiera y el régimen de tipo de cambio fijo resultaron ser incompatibles entre sí, y los ataques especulativos contra las monedas asiáticas y el rublo ruso obligaron al Brasil a adoptar un régimen de tipo de cambio flexible en enero de 1999. En junio de ese mismo año se implementaron nuevas disposiciones de política económica que incluían la fijación de objetivos de inflación, un superávit primario y un tipo de cambio flexible.

La reacción mexicana a la crisis de la deuda fue comenzar a revertir las políticas de intervención del Estado implementadas en la fase anterior. Así, la primera "fase de globalización" de la economía mexicana comenzó a mediados de la década de 1980, cuando comenzaron a implementarse diversas políticas de liberalización del comercio. En 1986, México adhirió al Acuerdo General sobre Aranceles Aduaneros y Comercio (GATT). El gobierno comenzó rápidamente a desmantelar el sistema de protección comercial, liberalizar el mercado financiero y reducir el sector público mediante la realización de privatizaciones y la disminución del gasto público. Lograr una inflación baja y constante se convirtió en el principal objetivo macroeconómico, pues se consideraba una condición necesaria y en gran medida suficiente para encaminar la economía hacia un crecimiento fuerte y duradero impulsado por las exportaciones e intensivo en mano de obra. Sin embargo, las políticas liberales no lograron los resultados esperados y la integración en la economía mundial se tradujo en un bajo crecimiento y una mayor dependencia de las exportaciones de petróleo. De hecho, entre 1982 y 1999 se registró el peor camino de crecimiento de la economía mexicana desde la Segunda Guerra Mundial. Una consecuencia inmediata de la adhesión de México al TLCAN en 1994 fue el desmantelamiento de las cadenas de producción del país, que abrió el paso a las maquilas. La especialización de la industria mexicana en sectores de alta tecnología condujo de hecho a la desindustrialización en la manufactura básica, que a su vez limitó el crecimiento de la demanda interna (Levy-Orlik, 2012, pág. 246). De acuerdo con Ibarra y Blecker (2014), además de la intensificación del proceso de desindustrialización, un ataque especulativo contra la moneda nacional en 1994 puso de manifiesto la limitada capacidad de la política interna para anclar la estabilización monetaria en un régimen de tipo de cambio fijo. La recuperación de la economía mexicana en función de su integración en las cadenas de suministro norteamericanas se observa en la fase sucesiva ${ }^{23}$.

\footnotetext{
${ }^{23}$ Desde que México se incorporó al mercado internacional mediante las cadenas mundiales de suministro, las empresas multinacionales han asumido un papel central en la producción. Así, el cambio estructural en el país durante la fase más aguda del liberalismo económico no impidió que se volviera financiera y tecnológicamente dependiente (principalmente de los Estados Unidos), aunque sí llevó a la diversificación y al aumento de la participación de la industria en la economía y del contenido tecnológico de las exportaciones (Levy-Orlik, 2012, pág. 237).
} 
Entre las cuatro economías estudiadas, la argentina fue la que presentó la peor trayectoria de crecimiento en la fase 1, mientras una serie de planes económicos cambió drásticamente su panorama económico en la fase 2. Como ya se mencionó, el movimiento hacia las políticas económicas liberales comenzó antes en la Argentina, cuando se estableció la plataforma económica liberal centrada en las políticas monetaristas. En 1982, la Argentina ocupó las Islas Malvinas y entró en conflicto con el Reino Unido. Esto tuvo como consecuencias una fuerte depreciación del peso, una grave inflación y la acumulación de una considerable deuda externa. En la década de 1980, las tasas de crecimiento fueron bajas y la inflación persistentemente alta se convirtió en un problema crónico, que se agravó debido a graves episodios de fuga de capitales hacia el final de la década. En 1991 se puso en marcha un controvertido plan de lucha contra la inflación, por el que el peso se volvió totalmente convertible con el dólar a un tipo de cambio fijo ${ }^{24}$. Aunque esto redujo fuertemente la inflación, el tipo de cambio fijo disminuyó el costo de las importaciones, provocando la fuga de dólares del país y una pérdida masiva de puestos de trabajo e infraestructura industrial. La recuperación de la economía argentina a principios de la década de 1990 estuvo asociada con la estabilización de la inflación y la apertura económica. Cunha y Ferrari (2009) afirman que, con la adopción del sistema de convertibilidad de la moneda en 1991, la Argentina llevó las políticas neoliberales al extremo. Si bien el programa de convertibilidad eliminó la hiperinflación, mostró poca capacidad para absorber los choques externos (Cunha y Ferrari, 2009, pág. 7). El tipo de cambio fijo fomentó la expansión del consumo privado, que se financió con un creciente endeudamiento externo. En un contexto de mayor inestabilidad en los mercados financieros internacionales durante la década de 1990, la Argentina se volvió cada vez más dependiente de los recursos oficiales, de los programas de medidas económicas (financial packages) liderados por el Fondo Monetario Internacional (FMI) y de la financiación del mercado de deuda privada. La insostenibilidad de este arreglo macroeconómico llegó a un punto crítico con la moratoria de 2001: mientras en diciembre de 1991 la deuda externa total de la Argentina era de 62.000 millones de dólares, equivalente al 32\% del PIB, en 2001 superaba los 140.000 millones de dólares, más del 50\% del PIB (Cunha y Ferrari, 2009, pág. 14).

La economía chilena era la más integrada en la década de 1980, pues el país había abandonado el modelo basado en la sustitución de importaciones a principios de la década de 1970. Sin embargo, al igual que el resto de las economías latinoamericanas, Chile sufrió una grave crisis externa tras la moratoria mexicana. En 1982, su moneda se devaluó considerablemente y llevó la economía a una fuerte recesión. El PIB se contrajo un 13,2\% en 1982 y un 2,8\% en 1983. Las autoridades económicas adoptaron una serie de medidas para atraer capital extranjero y gran parte de la deuda externa privada del país se convirtió en deuda externa pública como resultado de las intervenciones en el sistema financiero. Para reducir esta deuda, el gobierno optó por la conversión de deuda en capital, un mecanismo por el que ofrecía recomprar los bonos de los inversionistas extranjeros titulares de deuda chilena a la par, pero en pesos chilenos y con la condición de que el capital se reinvirtiera en el país. Años más tarde, cuando la economía se había estabilizado, se reconocieron los méritos de esta solución chilena a la crisis. La apertura económica y la temprana integración en la economía mundial hicieron que los recursos se reasignaran a sectores industriales orientados al mercado exterior (Carton y Slim, 2012). El relativo buen desempeño económico de Chile en la década de 1990, basado en la ampliación y diversificación de las exportaciones de recursos naturales, fue el resultado de las reformas estructurales de las décadas anteriores. De acuerdo con Díaz (2013, pág. 219), las políticas macroeconómicas liberales se consolidaron en los años noventa y fueron la base de la recuperación de la economía.

En resumen, las políticas de liberalización comercial y financiera se convirtieron en los pilares de la mayoría de las economías latinoamericanas, y de las cuatro economías líderes en particular, inaugurando decisivamente un nuevo patrón de crecimiento impulsado por las fuerzas del mercado.

\footnotetext{
${ }^{24}$ Cabe señalar que los primeros años de la convertibilidad fueron muy positivos en cuanto al crecimiento de los ingresos internos y el éxito en la lucha contra la inflación crónica (Ferrari y Cunha, 2008, pág. 50). Entre 1991 y 1998, la tasa media de crecimiento anual de la Argentina fue de alrededor del 6\%.
} 


\section{Fase 3: nuevo régimen de crecimiento basado en la integración económica en un mundo asimétrico (2000-2014)}

La tercera fase comenzó en la década de 2000 y constituyó una época de mayor integración en la economía mundial para las cuatro economías analizadas, en la que se recuperó el crecimiento de la productividad y la inversión por trabajador ${ }^{25}$.

El nuevo régimen de crecimiento de la integración económica fue el resultado de las políticas económicas orientadas al mercado implementadas en las décadas de 1980 y 1990. Estas contribuyeron a un cambio estructural que condujo a una mayor especialización en la producción de productos básicos y, en el caso de México, a la participación en las cadenas mundiales de suministro. Con el apoyo de las políticas económicas liberales, la Argentina, el Brasil, Chile y México experimentaron una reorganización industrial que permitió el aumento de las exportaciones desde la década de 1980 hasta 2000, aunque los efectos ingreso de la apreciación del tipo de cambio y la marcada dependencia de la industria de los insumos importados en todos los países hicieron que las importaciones crecieran aún más rápido (véase el cuadro 1). Incluso entre 2003 y 2007, el período de mayor crecimiento en América Latina desde la Segunda Guerra Mundial, ninguna de las cuatro economías estudiadas logró invertir la tendencia estructural y reducir la brecha tecnológica con las economías más desarrolladas.

En el caso del Brasil, la recuperación de la trayectoria de crecimiento a partir de 2000 puede considerarse débil. Si bien la trayectoria de crecimiento se encuentra en la parte de innovación del framework space, el crecimiento medio de la productividad fue menor que en la fase 1. Este resultado puede interpretarse como una incapacidad por parte de los responsables de la formulación de políticas de coordinar estrechamente las políticas de mejora de la productividad (como las políticas industriales, tecnológicas y comerciales) con las políticas macroeconómicas a corto plazo (especialmente las políticas monetarias y cambiarias) (Bresser-Pereira, Nassif y Feijo, 2016; Nassif, Bresser-Pereira y Feijo, 2018). En otras palabras, el régimen de política macroeconómica del Brasil, que combina un régimen de objetivos fiscales y de inflación con un régimen de tipo de cambio flotante, no ha logrado aumentar el espacio de políticas para las políticas de crecimiento. El modus operandi conservador de este "trípode" de la política macroeconómica brasileña no ha logrado reducir las tasas de interés internas a corto plazo ni cerca de los niveles internacionales ni evitar una tendencia cíclica a la sobrevaloración de la moneda brasileña en términos reales.

La orientación de la política macroeconómica de la economía mexicana intensificó la consolidación de las industrias exportadoras del país en la década de 2000. De hecho, las exportaciones de manufacturas se incrementaron en forma constante a tasas anuales superiores al 10\% hasta el estallido de la crisis financiera internacional en 2008 y 2009. De acuerdo con Moreno (2015), este logro está lejos de haberse traducido en un crecimiento elevado y sostenido, libre de crisis financieras o de la balanza de pagos. Esto se debe a que todas las iniciativas de la economía mexicana para integrarse en el TLCAN condujeron a la desnacionalización de la economía, pues la transferencia de tecnología e instalaciones de investigación y desarrollo de las empresas multinacionales a México fue muy escasa. El crecimiento del sector maquilador dio lugar a la desindustrialización. Según Ocampo (2015), todo esto ocurrió a pesar de un tipo de cambio real competitivo, al que se llegó mediante la represión salarial y políticas económicas contractivas. En el gráfico 2, México se posiciona en la parte de acumulación del framework space.

En el caso de la Argentina, la economía se sumió en una profunda depresión que duró desde 1999 hasta 2002, cuando el plan de convertibilidad resultó insostenible. La recuperación llegó a partir

\footnotetext{
${ }^{25}$ Carvalho (2008, pág. 122) cuestiona la resiliencia de las opciones liberales en vista de los problemas que la mayoría de las economías latinoamericanas enfrentó en la década de 1990. Según el autor, las profundas crisis de los años noventa no modificaron las principales características de los regímenes financieros creados en el proceso de liberalización.
} 
de 2003 con el gobierno de Kirchner y la implementación de políticas para sostener la demanda agregada. Sin embargo, como señala Porta (2015), a finales de 2007 la trayectoria de crecimiento mostraba signos de considerables desequilibrios, la mayoría de los cuales se arraigaba en la estructura productiva argentina. Este diagnóstico se basa en la evidencia de que la estructura productiva argentina está centrada en la producción de baja tecnología (Porta, 2015). Además, como señalan Cunha y Ferrari (2009), el proceso de recuperación económica de la década de 2000, que implicó un cambio de rumbo en la política macroeconómica con respecto al modelo neoliberal vigente hasta 2001-2002, no debe entenderse como un retorno a una estrategia de crecimiento desarrollista. Los autores sugieren que, en una perspectiva histórica de más largo plazo, el fin de la era Kirchner ha demostrado que la sociedad argentina sigue estando mucho más dispuesta a adherir al modelo liberal, en sus diferentes versiones, que a las estrategias de desarrollo que buscan cambiar estructuralmente la base productiva (Cunha y Ferrari, 2009, pág. 21).

La economía chilena es la única de las cuatro que puede considerarse beneficiada por la apertura económica y la especialización en recursos naturales. Sin embargo, la trayectoria de crecimiento del país no ha señalado un cambio de régimen de crecimiento desde la década de 1950. Si bien la capacidad de crecimiento y el dinamismo de las exportaciones de la economía chilena aumentaron, la apertura económica provocó una mayor inestabilidad. El aumento de las entradas de capital extranjero y el "mal holandés" asociado con las exportaciones de cobre han provocado un prolongado ciclo de apreciación del tipo de cambio real que afecta la competitividad de la producción y las exportaciones de bienes y servicios con mayor valor agregado. Así, la productividad de la estructura productiva es muy heterogénea y la economía chilena sigue siendo muy dependiente de las exportaciones de cobre. A pesar de que los indicadores macroeconómicos se han mantenido estables gracias a la implementación de políticas macroeconómicas consistentes desde la década de 1990, no se ha avanzado de la misma manera con los instrumentos de desarrollo o de política para promover el desarrollo económico (Díaz, 2013, págs. 246-252).

\section{Observaciones finales}

Del análisis comparativo de las trayectorias de crecimiento de la Argentina, el Brasil y México se puede concluir que el mal desempeño reciente de estas economías no debe considerarse como un fenómeno cíclico, sino como el resultado de la forma en que cada una se integró a la economía mundial. En todos los casos, los cambios de régimen de crecimiento desde la década de 1950 han significado la reducción del espacio de políticas y del potencial de crecimiento debido a una creciente vulnerabilidad externa.

Desde la primera fase (fase 1) hasta la última (fase 3), los cambios estructurales implicaron el desarrollo de industrias especializadas en productos básicos y manufacturados de baja tecnología (Argentina y Brasil) y de maquila de alta tecnología (México). Asimismo, el framework space muestra que el crecimiento de la productividad en las cuatro economías fue menor que en la fase 1. La Argentina mostró el patrón de crecimiento más inestable, pues su trayectoria de crecimiento se acercó al corredor harrodiano.

Los resultados de crecimiento a largo plazo de Chile son muy distintos con respecto a los de los demás países analizados pues, aunque todavía se basa esencialmente en los recursos naturales, ha mantenido el mismo patrón de crecimiento durante los últimos 50 años y ha logrado diversificar su estructura productiva.

Por otra parte, la apertura de estas economías antes de que pudieran alcanzar a las desarrolladas profundizó su dependencia de los flujos de capital internacionales. La larga transición de la fase 2 a la 3 , la fase de la integración económica modificó sustancialmente el papel del Estado, que se volvió menos intervencionista. Los inversionistas privados con acceso a los mercados financieros internacionales 
se convirtieron en los principales actores que guiaban las decisiones de inversión y la acumulación de capital. En este sentido, puede decirse que el compromiso con un enfoque desarrollista que prevalecía durante la fase de industrialización dirigida por el Estado cedió el paso a criterios de rentabilidad a corto plazo. Las políticas de estabilización se convirtieron en la prioridad de los responsables de la política económica y estas redujeron el espacio para las políticas económicas a largo plazo. La mayoría de las veces, su implementación se tradujo en un aumento de las tasas de interés real y una reducción de los tipos de cambio real, que desincentivaron la acumulación de capital en términos reales a largo plazo. Así, la especialización en la producción de bienes de escaso valor agregado y el aumento de la financierización son fenómenos que se produjeron conjuntamente después de que la apertura económica redujera el espacio de políticas.

Por último, el auge del comercio internacional en la década de 2000 "desató una ola de prosperidad para las economías en desarrollo y, en particular, para América Latina, que influenció sus estrategias de desarrollo e inserción externa” (De Souza y Ferraz, 2015, pág. 411). La crisis financiera internacional de 2008 provocó un cambio repentino en esta situación. Una pregunta que se plantea es si las economías semiindustrializadas objeto de este estudio están preparadas para el período de menor comercio internacional y mayor incertidumbre financiera derivados de la hasta ahora lenta recuperación de las economías desarrolladas. Esta pregunta también plantea el tema del margen de maniobra que se ha dejado a cada economía en las gestiones para sostener el crecimiento. Ahora que se han puesto en marcha políticas macroeconómicas orientadas al mercado y que el cambio estructural ha dado lugar a un desplazamiento hacia las industrias de baja tecnología, ¿están estas economías mejor situadas para hacer frente a una desaceleración internacional del comercio?

Un conjunto de recomendaciones de políticas destinadas a promover un cambio estructural hacia sectores más avanzados tecnológicamente debería prever una política macroeconómica coherente, capaz de ampliar el alcance de la política industrial para lograr los mejores resultados en términos de cambio económico dinámico. Las políticas macroeconómicas deberían ser anticíclicas y, en consecuencia, la gestión de los flujos de capital debería ser una opción para evitar la volatilidad del capital, que afecta negativamente los tipos de cambio nominal y real. Por último, la política industrial debería estar diseñada para permitir la toma de decisiones estratégicas sobre el desarrollo económico a largo plazo.

\section{Bibliografía}

Aghion, P. y P. Howitt (2009), The Economics of Growth, Cambridge, The MIT Press.

Bárcena, A. y A. Prado (eds.) (2015), Neoestructuralismo y corrientes heterodoxas en América Latina y el Caribe a inicios del siglo XXI, Libros de la CEPAL, № 132 (LC/G.2633-P/Rev.1), Santiago, Comisión Económica para América Latina y el Caribe (CEPAL), abril.

Bértola, L. y J. Ocampo (2012), The Economic Development of Latin America since Independence, Oxford, Oxford University Press.

Böhm, B. y L. Punzo (2001), "Productivity-investment fluctuations and structural change", Cycles, Growth and Structural Change: Theories and Empirical Evidence, L. Punzo (ed.), Londres, Routledge.

(1994), "Dynamics of industrial sectors and structural change in the Austrian and Italian economies, 1970-1989", Economic Performance: A Look at Austria and Italy, Berlín, Springer.

(1992), "Detecting structural change: a scheme for the comparison of Austria and Italy in the seventies and eighties", Technological Innovation, Competitiveness, and Economic Growth, O. Clauser y otros (eds.), Berlín, Duncker and Humboldt.

Bresser-Pereira, L., A. Nassif y C. Feijo (2016), "A reconstrução da indústria brasileira: a conexão entre o regime macroeconômico e a política industrial”, Revista de Economía Política, vol. 36, № 3, São Paulo, Centro de Economía Política.

Brida, J. y L. Punzo (2003), "Symbolic time series analysis and dynamic regimes", Structural Change and Economic Dynamics, vol. 14, № 2, Ámsterdam, Elsevier. 
Câmara, A. y M. Vernengo (2013), "Padrões de investimento, crescimento e produtividade na econoima argentina", Padrões de desenvolvimento econômico (1950-2008): América Latina, Ásia e Rússia, vol. 1, Brasilia, Centro de Gestión y Estudios Estratégicos (CGEE).

Carton, C. y S. Slim (2012), "Regional integration and growth: an empirical assessment for Latin American Countries", Beyond the Global Crisis: Structural Adjustments and Regional Integration in Europe and Latin America, L. Punzo, C. Feijó y M. Puchet (eds.), Milton Park, Routledge.

Carvalho, F. (2008), "Financial liberalization in Brazil and Argentina", Financial Liberalization and Economic Performance in Emerging Countries, P. Arestis y L. de Paula (eds.), Nueva York, Palgrave Macmillan.

Chang, H. J. (2003), Kicking Away the Ladder: Development Strategy in Historical Perspective, Londres, Anthem Press.

Cunha, A. y A. Ferrari (2009), "A Argentina depois da conversibilidade: um caso de novo-desenvolvimentismo?", Revista de Economia Política, vol. 29, № 1, São Paulo, Centro de Economía Política.

De Long, J. y L. Summers (1991), "Equipment investment and economic growth", The Quarterly Journal of Economics, vol. 106, № 2, Oxford, Oxford University Press, mayo.

De Souza, F. y J. Ferraz (2015), "¿Quo vadis, desarrollo brasileño?”, Neoestructuralismo y corrientes heterodoxas en América Latina y el Caribe a inicios del siglo XXI, Libros de la CEPAL, № 132 (LC/G.2633-P/Rev.1), A. Bárcena y A. Prado (eds.), Santiago, Comisión Económica para América Latina y el Caribe (CEPAL), abril.

Díaz, A. (2013), "Evolução e transformação estrutural da economia Chilena 1950-2009", Padrões de desenvolvimento econômico (1950-2008): América Latina, Ásia e Rússia, vol. 1, Brasilia, Centro de Gestión y Estudios Estratégicos (CGEE).

Dixon, R. y A. Thirlwall (1975), "A model of regional growth rate differences on Kaldorian lines", Oxford Economic Papers, vol 27, № 2, Oxford, Oxford University Press, julio.

Epstein, G. (ed.) (2005), Financialization and the World Economy, Cheltenham, Edward Elgar Publishing.

Feijo, C., L. Punzo y M. Lamônica (2012), "Brazil's economy - 1971-2008: growth pattern and structural change", Beyond the Global Crisis: Structural Adjustments and Regional Integration in Europe and Latin America, L. Punzo, C. Feijó y M. Puchet (eds.), Milton Park, Routledge.

Ferrari, A. y A. Cunha (2008), "As origens da crise argentina: uma sugestão de interpretação", Economia e Sociedade, vol. 17, № 2, Campinas, Universidad Estatal de Campinas, agosto.

Ferrer, A. (2004), La economía argentina: desde sus orígenes hasta principios del siglo XXI, Buenos Aires, Fondo de Cultura Económica (FCE).

Gaffard, J. y L. Punzo (2005), "Economic integration and cross-country convergence: exercises in growth theory and empirics", Inequality and Economic Integration, F. Farina y E. Savaglia (eds.), Londres, Routledge.

Ibarra, C. y R. Blecker (2014), "Structural change, the real exchange rate and the balance of payments in Mexico, 1960-2012", Cambridge Journal of Economics, vol. 40, № 2, Oxford, Oxford University Press.

Kaldor, N. (1957), "A model of economic growth", The Economic Journal, vol. 67, № 268, Oxford, Oxford University Press.

Kaldor, N. y J. Mirrlees (1962), "A new model of economic growth", The Review of Economic Studies, vol. 29, № 3, Oxford, Oxford University Press.

Kume, H., G. Piani y C. de Souza (2003), "A política brasileira de importação no período 1987-98: descrição e avaliação", A abertura comercial brasileira nos anos 1990: impactos sobre emprego e salário, C. Corseuil y H. Kume (eds.), Río de Janeiro, Instituto de Investigaciones Económicas Aplicadas (IPEA).

León-Ledesma, M. (2002), "Accumulation, innovation and catching-up: an extended cumulative growth model", Cambridge Journal of Economics, vol. 26, № 2, Oxford, Oxford University Press.

León-Ledesma, M. y A. Thirlwall (2002), "The endogeneity of natural rate of growth", Cambridge Journal of Economics, vol. 26, № 4, Oxford, Oxford University Press.

Levy-Orlik, N. (2012), "Effects of financialization on the structure of production and nonfinancial private enterprises: the case of Mexico", Journal of Post Keynesian Economics, vol. 35, № 2, Milton Park, Taylor and Francis.

Libânio, G. (2009), "Aggregate demand and the endogeneity of the natural rate of growth: evidence from Latin American economies", Cambridge Journal of Economics, vol. 33, № 5, Oxford, Oxford University Press, septiembre.

Moreno, J. (2015), "Desarrollo y macroeconomía: reflexiones a partir del caso mexicano", Neoestructuralismo y corrientes heterodoxas en América Latina y el Caribe a inicios del siglo XXI, Libros de la CEPAL, № 132 (LC/G.2633-P/Rev.1), A. Bárcena y A. Prado (eds.), Santiago, Comisión Económica para América Latina y el Caribe (CEPAL), abril. 
Moreno, J. y E. Pérez (2009), "Trade and economic growth: a Latin American perspective on rhetoric and reality", serie Estudios y Perspectivas, № 119 (LC/L.3179-P - LC/MEX/L.945), Santiago, Comisión Económica para América Latina y el Caribe (CEPAL), noviembre.

Nassif, A., L. Bresser-Pereira y C. Feijo (2018), "The case for reindustrialisation in developing countries: towards the connection between the macroeconomic regime and the industrial policy in Brazil", Cambridge Journal of Economics, vol. 42, № 2, Oxford, Oxford University Press, marzo.

Nassif, A., C. Feijo y E. Araújo (2016), "The BRICS growth economic performance before and after the international financial crisis", International Journal of Political Economy, vol 45, № 4, Milton Park, Taylor and Francis.

Ocampo, J. (2015), "América Latina frente a la turbulencia económica mundial", Neoestructuralismo y corrientes heterodoxas en América Latina y el Caribe a inicios del siglo XXI, Libros de la CEPAL, № 132 (LC/G.2633-P/Rev.1), A. Bárcena y A. Prado (eds.), Santiago, Comisión Económica para América Latina y el Caribe (CEPAL), abril.

(2007), "Instability and inequalities of the Global Reserve System", Working Papers, № 59, Nueva York, Naciones Unidas.

Ocampo, J. y R. Vos (2008), "Policy space and the changing paradigm in conducting macroeconomic policies in developing countries", New Financing Trends in Latin America: A Bumpy Road Towards Stability, BIS Papers, № 36, Basilea, Banco de Pagos Internacionales (BPI).

Porta, F. (2015), "Trayectorias de cambio estructural y enfoques de política industrial: una propuesta a partir del caso argentino", Neoestructuralismo y corrientes heterodoxas en América Latina y el Caribe a inicios del siglo XXI, Libros de la CEPAL, № 132 (LC/G.2633-P/Rev.1), A. Bárcena y A. Prado (eds.), Santiago, Comisión Económica para América Latina y el Caribe (CEPAL), abril.

Punzo, L. (2006), "Towards a disequilibrium theory of structural dynamics: Goodwin's contributions", Structural Change and Economic Dynamics, vol. 17, № 4, Ámsterdam, Elsevier.

Rey, H. (2015), "Dilemma not trilemma: the global financial cycle and monetary policy independence", Working Paper, № 21.162, Cambridge, Oficina Nacional de Investigaciones Económicas (NBER), mayo.

Romer, D. (2012), Advanced Macroeconomics, Nueva York, McGraw Hill.

Ros, J. (1993), "Mexico's trade and industrialization experience since 1960: a reconsideration of past policies and assessment of current reforms", Working Paper, № 186, Notre Dame, Kellogg Institute for International Studies, enero.

Sapelli, C. (2003), "The political economics of import substitution industrialization", Documentos de Trabajo, № 257, Santiago, Pontificia Universidad Católica de Chile (PUC).

Solow, R. (1957), "Technical change and the aggregate production function", The Review of Economics and Statistics, vol. 39, № 3, Cambridge, The MIT Press, agosto.

Syverson, C. (2010), "What determines productivity?", NBER Working Paper series, № 15.712, Cambridge, Oficina Nacional de Investigaciones Económicas, enero.

Thirlwall, A. (1979), "The balance of payments constraint as an explanation of international growth rate differences", BNL Quarterly Review, vol. 32, № 128, Roma, Banca Nazionale del Lavoro.

Titelman, D. y E. Pérez (2015), "Macroeconomía para el desarrollo en América Latina y el Caribe: nuevas consideraciones sobre las políticas anticíclicas", Neoestructuralismo y corrientes heterodoxas en América Latina y el Caribe a inicios del siglo XXI, Libros de la CEPAL, № 132 (LC/G.2633-P/Rev.1), A. Bárcena y A. Prado (eds.), Santiago, Comisión Económica para América Latina y el Caribe (CEPAL), abril. 


\section{Anexo A1}

Las variables del framework space (véase el cuadro A1.1) se construyen sobre la base de datos de Penn World Tables, versión 9.0, de la siguiente manera:

- gv es la tasa de crecimiento de la relación entre el PIB real a precios locales constantes (en millones de dólares de 2011) y el número de personas empleadas (en millones).

- gi es la tasa de crecimiento de la relación entre el acervo de capital a precios locales constantes de 2011 (en millones de dólares de 2011) y el número de personas empleadas (en millones).

\section{Cuadro A1.1}

Estimación de gu y gi como medias geométricas

\begin{tabular}{|c|c|c|c|c|c|c|c|c|}
\hline \multirow{2}{*}{ Fase } & \multicolumn{2}{|c|}{ Argentina } & \multicolumn{2}{|c|}{ Brasil } & \multicolumn{2}{|c|}{ Chile } & \multicolumn{2}{|c|}{ México } \\
\hline & $g v$ & $g i$ & $g v$ & $g i$ & $g v$ & $g i$ & $g v$ & $g i$ \\
\hline 1951-1981 & 1,5922 & 2,5927 & 3,70870 & 2,09340 & 2,1660 & 3,7082 & 3,18720 & 2,0081 \\
\hline 1982-1999 & 0,3963 & 0,5124 & 0,14949 & 1,069462 & 1,8098 & 2,7287 & $-1,0925$ & 0,1594 \\
\hline $2000-2014$ & 1,0488 & 0,8914 & 1,060694 & 0,721482 & 1,4214 & 2,9241 & 0,3756 & 1,7549 \\
\hline
\end{tabular}

Fuente: Elaboración propia, sobre la base de Universidad de Groninga, Penn World Tables 9.0 [base de datos en línea] https:// www.rug.nl/ggdc/productivity/pwt/pwt-releases/pwt9.0?lang=en. 